\title{
0 steonecrosis asociada al uso de bifosfonatos: A propósito de un caso clínico
}

\author{
Timoleón Anguita $C^{1}$, Jaime Agurto $\mathbf{P}^{2}$, Iván Roa $\mathrm{E}^{3}$, \\ German Laissle $\mathbf{C}^{2}$.
}

\section{O steonecrosis associated with the use of biphosphonates: Case report}

Biphosphonates reduce the risk of skeletal events and are currently part of standards of therapy in myeloma. Recently, zoledronate and pamidronate have been linked to osteonecrosis of the jaw, specially after surgical dental procedures. We report a 84 year-old man with multiple myeloma who developed spontaneous osteonecrosis of both jaws, after 36 months of therapy with zoledronate with a cumulative dose of $136 \mathrm{mg}$. We discuss the pathogenic mechanisms, and review the recommendations on prevention and management of this new complication for neoplastic patients under prolonged therapy with biphosphonates (Rev Méd Chile 2006; 134: 1161-5).

(Key w ords: Diphosphonates; Myeloma; Zoledronic acid)

Recibido el 5 de diciembre, 2005. Aceptado el 24 de marzo, 2006.

Departamento de Oncohematología1, Servicio de Cirugía Maxilofacial ${ }^{2}$, Servicio de Anatomía Patológica ${ }^{3}$, Clínica Alemana de Santiago.

$\mathrm{D}$ esde 2003, se han reportado casos de osteonecrosis maxilar asociada al uso de bifosfonatos como pamidronato y zoledronato, en pacientes con malignidades como mieloma, cáncer de mama, testículo, pulmón, próstata, sarcoma uterino y leucemia mieloide crónica postransplante de precursores hematopoyéticos y en osteoporosis sin malignidad, por ibandronato $\mathrm{y}$ risedronato ${ }^{1,2}$. Más raramente, se ha reportado necrosis de otras áreas óseas en linfoma ${ }^{3}$. Entre estos diagnósticos, el mieloma y el cáncer de

Correspondencia a: Dr. Timoleón Anguita C. Vitacura 5951. Vitacura, Santiago. Fax: 56/2/ 2101153. Tel: 56/2/ 2101006. E mail: tanguita@alemana.cl. mama son los más frecuentemente reportados, por lo que la International Myeloma Foundation realizó una encuesta por Internet en 2004, con 1.203 respuestas que cubrían 904 casos de mieloma y 299 de cáncer de mama. Se comunicaron 62 casos de osteonecrosis confirmada y 54 casos con sospecha de ello en mieloma y, 13 casos confirmados y 23 con sospecha en cáncer de mama, en un total de 152 pacientes ${ }^{4}$.

Entre los casos de mieloma, $71 \%$ recibían zoledronato y $29 \%$ pamidronato, constituyendo una incidencia de $10 \%$ en 211 pacientes para zoledronato y $4 \%$ en 413 pacientes para pamidronato, a los 36 meses de tratamiento ${ }^{4}$.

El uso de bifosfonatos ha constituido un gran aporte al control de la enfermedad ósea del mieloma, donde es parte del tratamiento estándar, 
y también en el cáncer de mama metastático ${ }^{5-7}$. La posibilidad de esta complicación, no debe alejar su uso, sino más bien obliga a definir el riesgo y conocer su patogenia, para establecer métodos de prevención y tratamiento. El objetivo de esta comunicación es presentar un caso de osteonecrosis bimaxilar en mieloma múltiple, asociado al uso de bifosfonatos.

\section{CASO CLÍNICO}

Paciente hombre de 80 años que ingresó a Clínica Alemana en abril de 2000, con diagnóstico de neumonía, siendo tratado, con evolución satisfactoria. En su estudio, se detectó paraproteína IgG kappa de 3,2 g/dL con hipogamaglobulinemia de las inmunoglobulinas normales. Sin embargo, no se demostraron criterios diagnósticos de enfermedad neoplásica activa: radiografías óseas sin osteolisis, función renal y examen de orina normal, sin cadenas livianas en orina por inmunofijación, mielograma con $7 \%$ de serie plasmática, perfil bioquímico normal, calcemia normal, creatinina y hemograma normal, con VHS de $100 \mathrm{~mm} / \mathrm{h}$, beta 2 microglobulina normal y proteína $\mathrm{C}$ reactiva normal.

Como gamopatía monoclonal de significado incierto, se indicó control periódico, observándose estabilidad de la paraproteína hasta julio de 2000, en que abandona controles.

En agosto de 2001, acudió a consulta, apreciándose aumento de la paraproteína a $4 \mathrm{~g} / \mathrm{dL}$, lesiones osteolíticas de trocánter mayor izquierdo y diáfisis femorales, aplastamiento en cuña de L3, L4 y L5, con osteopenia difusa, anemia de $9 \mathrm{~g} / \mathrm{dL} \mathrm{Hb}$, mielograma con $18 \%$ de serie plasmática, función renal normal, examen de orina normal, ausencia de proteína Bence-Jones por inmunofijación; perfil bioquímico, calcemia, beta 2 microglobulina y proteína $\mathrm{C}$ reactiva normales.

Se hizo diagnóstico de mieloma IgG kappa, Bence-Jones (-) estadio IIIA de Durie Salmon, e inició tratamiento con thalidomide $100 \mathrm{mg} /$ día, dexametasona $40 \mathrm{mg} /$ día por 4 días cada mes, transfusiones de glóbulos rojos y luego eritropoyetina $6.000 \mathrm{U} 3$ veces por semana, ácido zoledrónico $4 \mathrm{mg}$ al mes, iv, en $50 \mathrm{~mL}$ de suero fisiológico en $15 \mathrm{~min}$. La paraproteína disminuyó a 0,6 gr/ dL, manteniéndose hasta febrero de 2003. Se reduce la thalidomide a $50 \mathrm{mg} /$ día y recibió sólo irregularmente dexametasona, debido a efectos secundarios como inestabilidad de la marcha por fatigabilidad muscular.

Se asistió entonces a un aumento de la paraproteína a $2 \mathrm{~g} / \mathrm{dL}$ en marzo de 2004 y reaparición de la anemia, debiendo cambiarse tratamiento a melfalan, prednisona y transfusiones, lográndose estabilidad de la paraproteína y moderada mejoría de la fatigabilidad. En su evolución presentó progresivo aplastamiento, silencioso, de varias vértebras, con disminución de $15 \mathrm{~cm}$ de estatura.

En agosto de 2004, consultó en el Servicio Cirugía Maxilofacial, derivado por su dentista. Se constató exposición espontánea de tejido óseo por cara vestibular en ambos maxilares, en relación a piezas 18: segundo molar inferior izquierdo, 19: primer molar inferior izquierdo, 12: primer premolar superior izquierdo y 13: segundo premolar superior izquierdo. El hueso expuesto se observaba amarillento, de aspecto necrótico, sin fístulas, indoloro a la exploración, sin signos de osteomielitis y las piezas dentarias adyacentes presentaban movilidad acentuada. La tomografía axial computarizada no reveló evidencias de secuestración ósea. Se diagnosticó osteonecrosis avascular de ambos maxilares. La biopsia confirmó el diagnóstico, al constatar trabéculas óseas desvitalizadas, rodeadas por tejido granulatorio (Figura 1).

En septiembre de 2004 se realiza exodoncia de piezas 18, 19 y 12, esperando la delimitación espontánea del proceso, manteniendo aseo, irrigación con clorhexidina $0,12 \%$ y tratamiento antibiótico (amoxicilina/ácido clavulámico y doxiciclina). Frente a los antecedentes de asociación entre osteonecrosis maxilar y uso de zoledronato, éste se suspendió. El aspecto de la lesión, se observa en las Figuras 2 y 3.

En marzo de 2005 ocurrió la avulsión espontánea de la pieza 11. No se usó cámara hiperbárica, por lo discutible de su eficacia. En control semanal, hasta noviembre de 2005, no había presentado cambios, aunque el área ósea expuesta se había cubierto parcialmente por la encía.

Las dosis de zoledronato (4 $\mathrm{mg}$ cada una) recibidas desde el inicio del tratamiento fueron 34 , acumulando $136 \mathrm{mg}$ en un período de 36 meses hasta la aparición de los síntomas. 
Figura 1. Tejido óseo esponjoso, necrótico, rodeado por tejido granulatorio en organización (HE x 400).
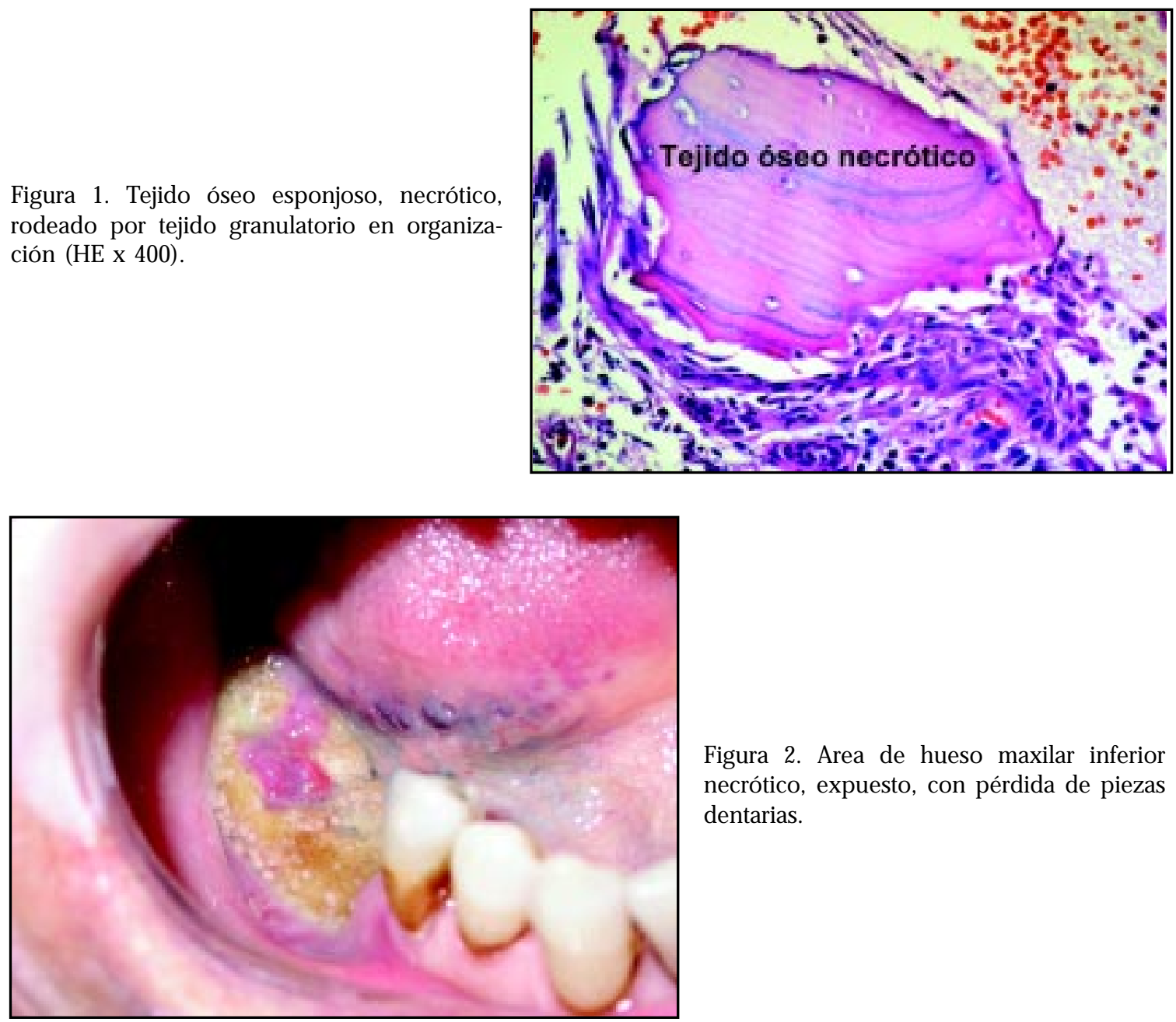

Figura 2. Area de hueso maxilar inferior necrótico, expuesto, con pérdida de piezas dentarias.

Figura 3. Area de hueso maxilar superior expuesto, necrótico, con pérdida de piezas dentarias.

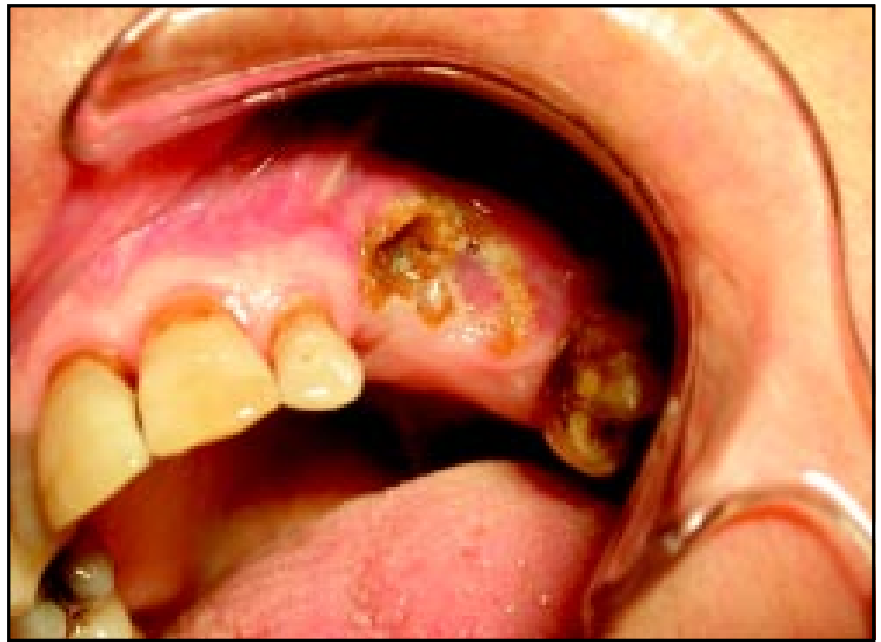




\section{DisCUSIÓN}

Los bifosfonatos son potentes inhibidores de los osteoclastos, tienen efecto antiangiogénico y antitumoral, con una vida media de años. Usados principalmente en mieloma múltiple y en metástasis óseas de tumores sólidos como el cáncer de mama, reducen los eventos óseos significativamente ${ }^{5-7}$. La asociación con osteonecrosis maxilar, sin embargo, no se ha establecido como una relación causa efecto, pero la incidencia en tiempos anteriores al uso de bifosfonatos era muy baja ${ }^{2,8}$, en cambio alcanza a $10 \%$ o más en las patologías mencionadas tratadas con zoledronato y algo menos con otros ${ }^{4}$.

Como explicación de la frecuente localización en maxilares, se ha invocado el estrés fisiológico a que son sometidas dichas áreas, únicas en exponer hueso al medio externo (vía dientes). Este estrés aumenta por procesos patológicos dentales y periodontales: abscesos, iatrogenia (tratamiento de conducto, extracciones u otros), que exigen aumentar la velocidad de recambio o remodelación ósea, la que se encuentra bloqueada por dichas drogas ${ }^{8-10}$. A esto se agrega el efecto antiangiogénico de los bifosfonatos, que provocan disminución del factor de crecimiento de endotelios $^{2,11}$, posiblemente explicando la mayor frecuencia de compromiso de la mandíbula (63-80\%), hueso de menor irrigación en relación al maxilar superior (14-38\%) ${ }^{1,2}$. Otros tratamientos antiangiogénicos como la thalidomide y los glucocorticoides, o de enfermedades concomitantes como diabetes mellitus, efectos de radioterapia, etc, han sido invocados como coadyuvantes ${ }^{1,8,9}$, sin embargo, en la encuesta realizada por la International Myeloma Foundation, los dos primeros no se demostraron como factores de ries$\mathrm{go}^{4}$.

La mayoría de los casos de osteonecrosis se presentan posterior a una extracción dental (78\%), infección u otro proceso y sólo una minoría (14\%) aparece espontáneamente. Sólo 5,5\% se presentan en ambos maxilares ${ }^{1,2}$. En nuestro paciente, el proceso apareció espontáneamente en ambos maxilares.

Asimismo, el plazo de aparición va desde 4 meses del inicio del tratamiento, hasta alcanzar $10 \%$ de los pacientes tratados al cabo de 36 meses ${ }^{4}$. La dosis acumulativa media en la publicación de Maerevoet y cols, fue de $72 \mathrm{mg}$ a los 18 meses $^{9}$. En nuestro caso, se presentó a los 36 meses del uso de zoledronato, habiendo acumulado $136 \mathrm{mg}$, lo que sugiere que la incidencia tiende a aumentar con el tiempo de uso y la dosis acumulada, como han observado otros $4,10,11$.

Para pacientes con los factores de riesgo mencionados, se han comunicado las siguientes recomendaciones en la prevención: revisión dental previa al inicio de los bifosfonatos, seguimiento y tratamiento de cualquier patología dentomaxilar, extracción de restos radiculares, tratamiento de la enfermedad periodontal, obturación de caries, con el mismo criterio recomendado para pacientes candidatos a radioterapia ${ }^{8}$. Higiene dental con antisépticos locales durante la terapia ${ }^{9}$. Realizar imágenes radiológicas ante cualquier síntoma local ${ }^{12}$. Evitar cirugía dental (extracciones, implantes, debridaciones, etc) durante la terapia ${ }^{1,5,13}$. Suspender bifosfonatos 3 meses antes de procedimientos dentales que se consideren imprescindibles durante la terapia ${ }^{14}$. Evaluar la necesidad de continuar la terapia más allá de 2 años y no iniciarla en mielomas indolentes sin osteopenia donde su utilidad no ha sido demostrada ${ }^{12}$.

En el manejo de la osteonecrosis establecida las recomendaciones son: suspensión del bifosfonato, higienización oral con clorhexidina 0,12\% en áreas óseas expuestas y especialmente en la zona de inserción mucoperióstica, que es propicia a la colonización bacteriana. Extracción de dientes desvitalizados comprometidos en el área de osteonecrosis. En caso de molestia lingual por contacto con áreas óseas o en caso de infección secundaria persistente, puede practicarse ostectomías limitadas. Cobertura antibiótica para evitar infección secundaria. Excepcionalmente, puede recurrirse a tratamientos cruentos: p.ej: una fractura mandibular puede requerir resección del segmento comprometido y reconstrucción con injerto libre o microvascularizado, estabilizándolo con placas de osteosíntesis o de reconstrucción mandibular. El uso de oxigenoterapia en cámara hiperbárica, para mejorar la oxigenación local, no se ha demostrado uniformemente efi$\mathrm{caz}^{15}$. 


\section{CONCLUSIÓN}

Este caso ejemplariza la osteonecrosis maxilar recientemente asociada al uso prolongado de bifosfonatos. La importante reducción de los eventos óseos en mieloma y cáncer de mama

\section{REFERENCIAS}

1. MARX RE. Pamidronate (Aredia) and Zoledronate (Zometa) induced avascular necrosis of the jaws: A growing epidemic. J Oral Maxilofac Surg 2003; 61: 1115-7.

2. Vitte C, Fleisch H, Guentes HL. Bisphosphonates induce osteoblasts to secrete an inhibitor of osteoclastic mediated resorption. Endocrinology 1996; 137: 2324-33.

3. Hughes DE, Mac Donald BR, Russell RG, Gowen M. Inhibition of osteoclastic-like cell formation by bisphosphonates in long-term cultures of human bone marrow. J Clin Invest 1989; 83: 1930-5.

4. DuRIE B. Osteonecrosis of the jaw and bisphosphonates (letter). N Engl J Med 2005; 353: 99-100.

5. Berenson JR, Lichtenstein A, Porter L, Dinopoulos MA, Bordony R, George S et al. Efficacy of pamidronate in reducing skeletal events in patients with advanced multiple myeloma. N Engl J Med 1996; 334: 448-93.

6. Theriault RL, Lipton A, Hortobagyi ON, LefF R, Gluck S, Stewart JF et al. Pamidronate reduces skeletal morbidity in women with advanced breast cancer and lytic lesions: A randomized placebocontrolled trial. J Clin Oncol 1999; 17: 846-54.

7. Berenson JR, Rosen LS, Howell A, Porter L, Coleman R, Morley W et al. Zoledronic acid reduces skeletal-related events in patients with osteolytic metastases. Cancer 2001; 91: 1191-200. metastático que aportan, los hace necesarios. Deben ser usados con precaución, especialmente en aspectos preventivos, así como en el manejo de la lesión establecida, gracias a una estrecha colaboración entre médicos y odontólogos.

8. Sook-Bin W, Hande K, Richardson PG. (letter). N Eng J Med 2005; 353: 100.

9. Maerevoet M, Martin C, Duck L. (letter). N Eng J Med, 2005; 353: 100-1.

10. Santini D, Vincenzi B, Avvisati G, Dicuonzo G, Battistoni F, Gavasci M et al. Pamidronate induces modifications of circulating angiogenic factors in cancer patients. Clin Cancer Res 2002; 8: 1080-4.

11. Durie BGM, Katz M, McCoy J, Crowley J. Osteonecrosis of the jaws in myeloma: Analysis of risk factors including time dependency of Aredia and Zometa use, steroid use and underlying dental problems. Haematologica/Hem Journal 2005; 90(s1): 190.

12. Singal S, Kut V, Tariman J, Rosen S, Bennet CL, MEHTA J. Pamidronate and Zoledronate-Associated osteonecrosis in myeloma is an increasing and under- recognized problem. Haematologica/Hem Journal 2005; 90(s1): 191.

13. Tarassoff P, Yong-Jiang Hei. (letter) Reply from Novartis. N Engl J Med 2005; 353: 101-2.

14. Thakkar SG, Isada C, Smith J, Karam MA, Reed J, TOMFORD JW ET AL. Bisphosphonate therapy and increased incidence of mandibular/maxillary osteomyelitis. Haematologica Hem Journal 2005; 90(s1): 191.

15. Ruggiero SL, Bhoomi Mhrotra, Rosemberg T, EmgroFF SL Osteonecrosis of the jaws associated with the use of biphosphonates: a review of 63 cases. J Oral Maxillofac Surg 2004; 62: 527-34. 